\title{
$\beta$ Mangostin suppress LPS-induced inflammatory response in RAW 264.7 macrophages in vitro and carrageenan-induced peritonitis in vivo
}

\begin{abstract}
Ethnopharmacological relevance: The fruit hull of Garcinia mangostana Linn. has been used in traditional medicine for treatment of various inflammatory diseases. Hence, this study aims to investigate the in vitro and in vivo anti-inflammatory effect of $\beta$ mangostin $(\beta \mathrm{M})$, a major compound present in Garcinia mangostana. Materials and methods: The in silico analysis of inflammatory mediators such as cyclooxygenase (COX) and nuclear factor-kappa B (NF-kB) were performed via molecular docking. Further evaluation of anti-inflammatory effect was conducted in lipopolysaccharide (LPS) induced RAW 264.7 macrophages. Suppression of activated NF-kB was analyzed by high content screening. $\beta \mathrm{M}$ triggered inhibition of COX-1 and COX-2 in vitro were studied using biochemical kit. The in vivo model used in this study was carrageenan-induced peritonitis model, where reduction in carrageenan-induced peritonitis is measured by leukocyte migration and vascular permeability. In addition, the evaluation of $\beta \mathrm{M}$ 's effect on carrageenan induced TNF- $\alpha$ and IL- $1 \beta$ release on peritoneal fluid was also carried out. Results: Treatment with $\beta \mathrm{M}$ could inhibit the LPS-induced NO production but not the viability of RAW 264.7. Similarly, $\beta$ M inhibited PGE2 production and the cytokines: TNF- $\alpha$ and IL-6. The COX catalyzed prostaglandin biosynthesis assay had showed selective COX-2 inhibition with a $53.0 \pm 6.01 \%$ inhibition at $20 \mu \mathrm{g} / \mathrm{ml}$. Apart from this, $\beta \mathrm{M}$ was capable in repressing translocation of NF-kB into the nucleus. These results were concurrent with molecular docking which revealed COX-2 selectivity and NF-kB inhibition. The in vivo analysis showed that after four hours of peritonitis, $\beta \mathrm{M}$ was unable to reduce vascular permeability, yet could decrease the total leukocyte migration; particularly, neutrophils. Meanwhile, dexamethasone $0.5 \mathrm{mg} / \mathrm{kg}$, successfully reduced vascular permeability. The levels of TNF- $\alpha$ and IL- $1 \beta$ in peritoneal fluid was reduced significantly by $\beta \mathrm{M}$ treatment. Conclusion: The current study supports the traditional use of Garcinia mangostana fruit hull for treatment of inflammatory conditions. In addition, it is clear that the anti-inflammatory efficacy of this plant is not limited to the presence of $\alpha$ and $\gamma$, but $\beta$ also with significant activity.
\end{abstract}

Keyword: $\beta$ Mangostin; Garcinia mangostana; iNOS; NF-kB; COX-2; Cytokines 